\title{
Assessment of Epidermal Barrier Function by Photoacoustic Spectrometry in Relation to Its Importance in the Pathogenesis of Atopic Dermatitis
}

\author{
Maki Hata, Yoshiki Tokura, Masahiro Takigawa, Makoto Sato, Yasushi Shioya, \\ Yoshiaki Fujikura, and Genji Imokawa
}

Department of Dermatology (MH, YT, MT), Hamamatsu University School of Medicine, Hamamatsu, and Kao Research Laboratories (MS, YS, YF, GI), Tochigi, Japan

SUMMARY: With the use of the photoacoustic spectrometry system, in which a mixture of lipid- and water-soluble dyes is applied to the skin and then irradiated with light from a xenon lamp (425 nm and $550 \mathrm{~nm}$ ), we measured photoacoustic signals of both dyes within the stratum corneum and their disappearance rate through the stratum corneum. The signal intensity was higher and dyes penetrated faster in clinically normal skin of patients with atopic dermatitis (AD) compared with healthy subjects, indicating an impairment of the in vivo cutaneous permeability barrier function against both lipophilic and hydrophilic chemicals. Furthermore, penetration rates of the hydrophilic dyes tended to increase in proportion to the severity of $A D$ and significantly correlated with serum IgE levels in the severe AD group. Thus, abnormal barrier functions of clinically normal skin in AD may predispose inflammatory processes evoked by irritants and allergens, especially their water-soluble elements. (Lab Invest 2002, 82:1451-1461).

$$
T
$$
here is a general consensus that disruption of the barrier function of the stratum corneum is an essential etiologic factor for skin inflammation in patients with atopic dermatitis (AD) (Gloor et al, 1981; Ogawa and Yoshiike, 1993; Tupker et al, 1990). Dysfunction of the stratum corneum seems to be associated with the increased permeability and penetration of environmental irritants and allergens in clinically normal AD skin (Cooper, 1994; Deleuran et al, 1998; Fitzharris and Riley, 1999; Gloor et al, 1981; Ogawa and Yoshiike, 1993; Rothe and Grant-Kels, 1996; Tan et al, 1996; Tanaka et al, 1994; Tupker et al, 1990), which predisposes it to inflammation (Broberg et al, 1992; Lammintausta et al, 1993). The impaired barrier function of the stratum corneum in AD has been found to result from the decreased production of ceramides (Imokawa et al, 1991, 1994), which are generated from sphingomyelin by sphingomyelinase (Hara et al, 2000; Higuchi et al, 2000; Murata et al, 1996; Wood et al, 1996). However, the activity of sphingomyelin deacylase, which hydrolyzes sphingomyelin at the acyl site to yield sphingosylphosphorylcholine, is abnormally high in clinically normal and lesional skin in AD (Hara et al, 2000), resulting in the ceramide deficiency (Imokawa et al, 1991). The defect of the barrier function in AD skin has been generally evidenced by an

DOI: 10.1097/01.LAB.0000036874.83540.2B

Received April 30, 2002

Address reprint requests to: Dr. Genji Imokawa, Kao Biological Science Laboratories, 2606 Akabane, Ichikai-machi, Haga, Tochigi 321-34 Japan.E-mail:imokawag@dream.ocn.ne.jp increased transepidermal water loss (TEWL) measured with an evaporimeter (which evaluates the evaporation rate of water through the skin) (Watanabe et al, 1991). Available data demonstrate a close correlation between the values of TEWL and the penetration of chemicals through the skin in vitro (Imokawa, 1993). It should be noted that the diffusion of evaporated water molecules through the stratum corneum represents only a facet of the barrier function and does not necessarily correlate with the diffusion of protein and chemicals that often affect inflammatory processes of AD. Re-evaluation of the barrier function of the stratum corneum on the basis of its in vivo permeability against reagents with different water solubilities may provide us further insights into the pathogenesis of AD. However, the lack of adequate instruments has hampered such study.

We have developed a new method, designated here as photoacoustic spectrometry (PAS) (Shioya et al, 2001), in which the percutaneous absorption of chemicals is evaluated by measuring PA signal changes over time. Those signals correspond to the amount of chemicals remaining in or penetrating the stratum corneum at a detection depth of approximately $14 \mu \mathrm{m}$. The reliability of this method for the in vivo measurement of the permeability of chemicals into the skin of normal individuals has already been established elsewhere (Takamoto et al, 1992, 1994). To ascertain the feasibility of our approach to measure the barrier function, it is important to apply the PAS method to skin with disrupted barrier function such as occurs in AD. In the present study, we determined the barrier function of the stratum corneum in normal individuals 
and in patients with $A D$. We demonstrate that PAS provides measurements of the barrier function distinct from those related to water evaporation. Comparison of the barrier function in normal individuals and in patients with $A D$ suggests that the abnormal barrier function of clinically normal skin in AD as measured by PAS predisposes the skin to allergic inflammation in response to a variety of environmental irritants and allergens.

\section{Results}

\section{The PA Signal, TEWL, and the Water-Holding Capacity in Human Skin}

We used both lipophilic (Red 215) and hydrophilic (Yellow 4) dyes as penetrants because different aspects of the barrier function of the skin could be obtained. A time course study demonstrated that, after topical application of each dye solution, the intensity of PA signals obtained with Red 215 dye (lipophilic) or with Yellow 4 dye (hydrophilic) decreased in a time-dependent manner in healthy control skin, with the rate of decrease for the former being less than the latter (Fig. 1A). In clinically normal skin of patients with $A D$, there was a similar time-dependent decrease in the intensity of PA signals obtained with Red 215 or Yellow 4 (Fig. 1B). However, the rates of decreases for both reagents in clinically normal skin in $A D$ were faster than in the healthy control skin, which suggests an impairment of the permeability barrier function in the clinically normal skin of $A D$ patients. Consistent with our previous report for patients with AD (Imokawa, 1999), clinically normal skin in AD exhibited a significant increase $(p<0.05)$ in TEWL

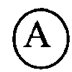

Healthy control

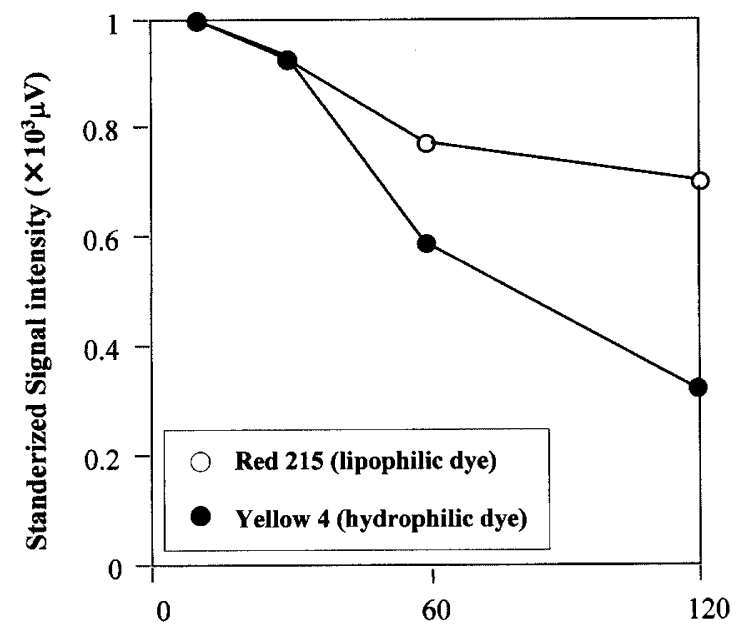

Time ( min)
(Fig. 2A) and a significant decrease $(p<0.05)$ in the water-holding capacity compared with healthy controls (Fig. 2B).

\section{Distribution of Dyes Penetrating the Stratum Corneum}

We then determined the distribution and penetration rate of dyes within the stratum corneum in two ways. First, after the dye solution was applied to the forearm skin for 2 minutes, the PA signal was measured at each of 15 tape stripping steps. In the healthy control and in the clinically normal skin in $A D$, the PA signals for both dyes decreased in proportion to the number of tape strippings. However, the signals became undetectable after 9 tape strippings in the healthy controls (Fig. 3A) but were still detectable even after 13 tape strippings in AD (Fig. 3B). In addition, the PA signal for Yellow 4 before tape stripping was higher in the clinically normal skin in $A D$ than in the healthy control. Next, PAS analysis after application of dyes for 2 or 5 minutes under the closed patch condition revealed that there was a significant increase in the PA signals under both application conditions for the lipophilic (Red 215) dye ( $n=100-103, p<0.001$; Fig. $4, \mathrm{~A}$ and $\mathrm{B}$ ) and for the hydrophilic (Yellow 4$)$ dye ( $n=$ 100-103, $p<0.001$; Fig. 4, C and D) through the stratum corneum in clinically normal skin in AD compared with healthy controls $(n=10)$. These data indicate that the PA signals measured in this study reflect concentrations of dyes penetrating the stratum corneum and that the clinically normal skin of patients with $A D$ has accelerated penetration rates for both types of dyes compared with healthy control skin.
(B)

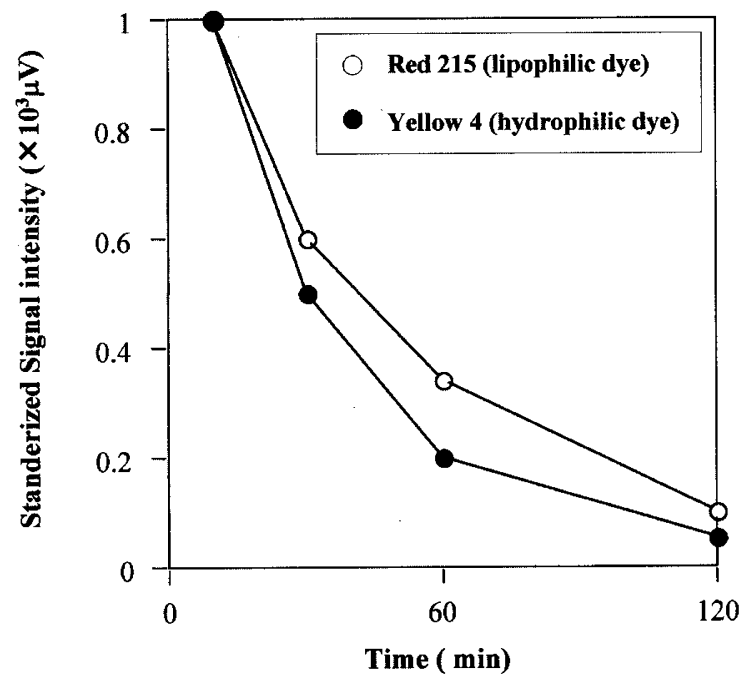

Figure 1.

Penetration profile of lipophilic and hydrophilic dyes through the stratum corneum of forearm skin. Red 215 and Yellow 4 were solubilized in $95 \%$ ethanol and $5 \%$ water at concentrations of 0.22 and $0.15 \mathrm{~mm}$, respectively. Each solution $(64 \mu \mathrm{l})$ was topically applied to the forearm skin. Thirty seconds later, the portable open-ended photoacoustic (PA) cell was attached to the skin surface of the treated-sites for subsequent measurements. A, Healthy control subject (28 years old, male). $B$, Patient with atopic dermatitis (AD) (26 years old, female). 
(A)

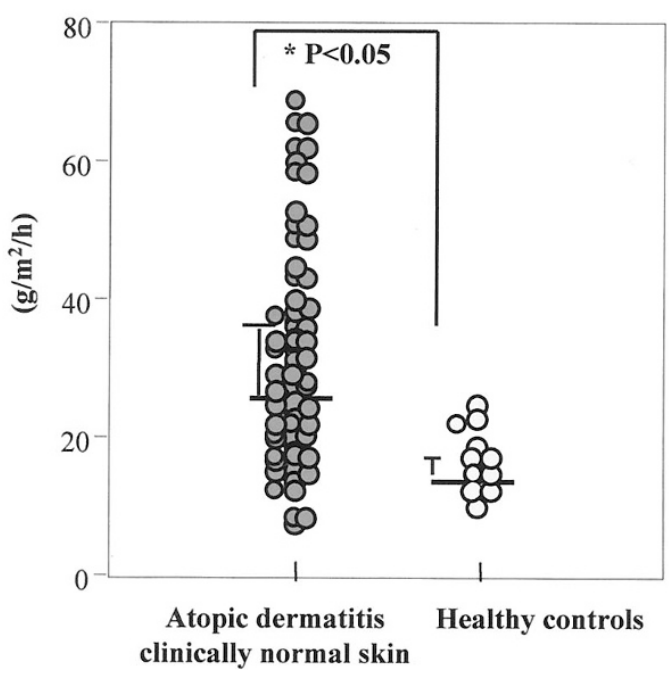

(B) B) Water-Holding Function

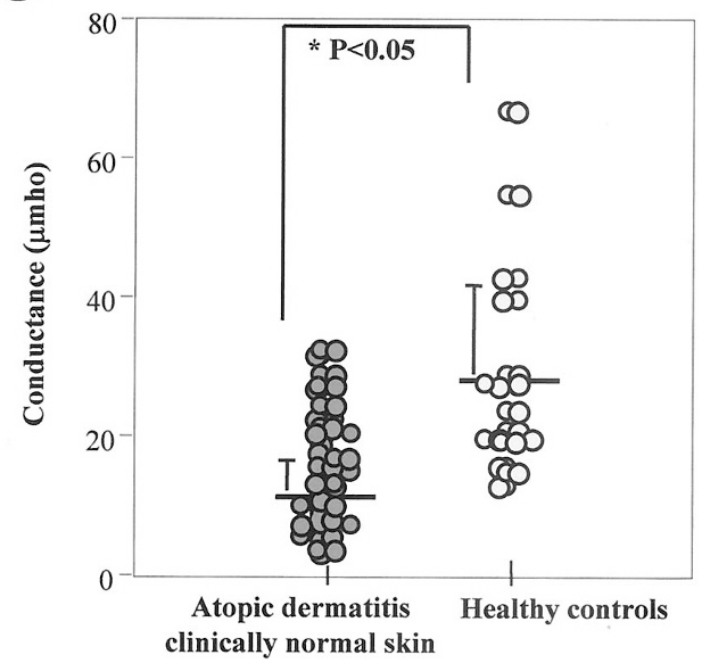

Figure 2.

Transepidermal water loss (TEWL) $(A)$ and water-holding function (B) of the uninvolved skin of patients with $A D$. TEWL from the skin was measured in $A D$ patients ( $n=104$, average age $=26.7$ years for $\mathrm{AD} ; n=18$, average age $=34$ years for control) by detecting evaporated water according to a modified method using a crystal vibration microbalance system (Endo et al, 2001). The water-holding function was assessed by an impedance meter $(n=86$, average age $=28$ years for $\mathrm{AD} ; n=16$, average age $=37$ years for control).

(A)

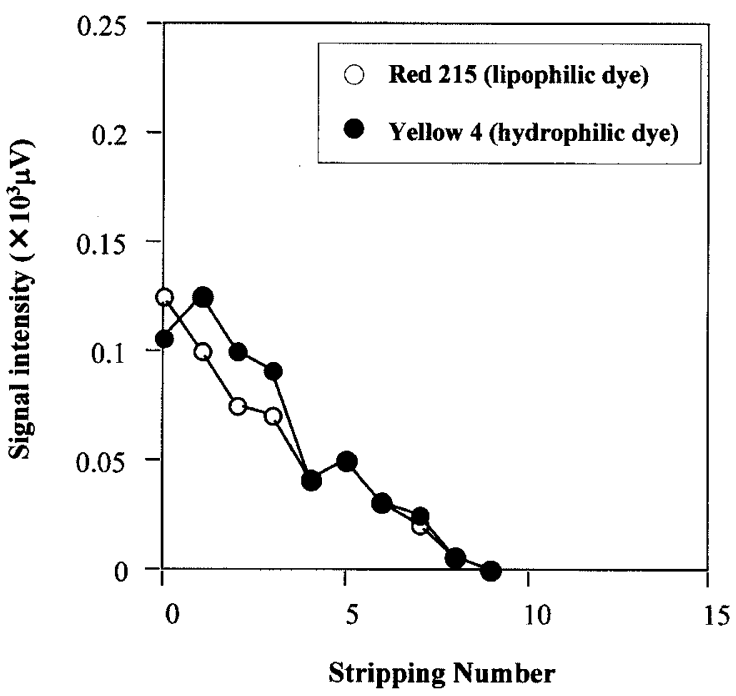

(B)

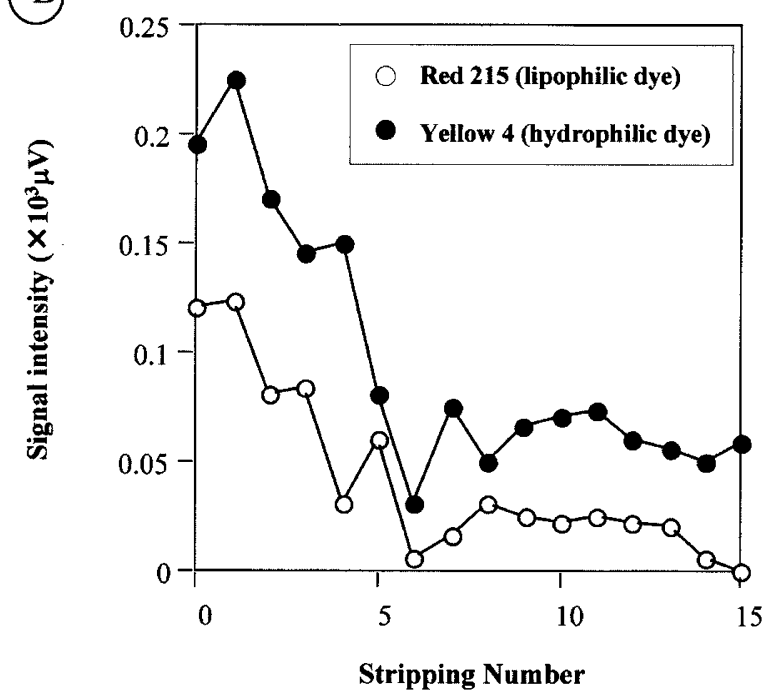

Figure 3.

Distribution of dye concentrations penetrating the stratum corneum as assessed by tape stripping. Solutions containing Red 215 and Yellow 4 in $95 \%$ ethanol at 0.22 $\mathrm{mm}$ and $0.15 \mathrm{~mm}$, respectively, were applied for 2 minutes to the forearm skin. PA signals were measured by the photoacoustic system (PAS) following each step of tape stripping, repeated 15 times. A, Healthy control subject (26 years old, male). B, Patient with AD (24 years old, male).

\section{Relationship Between the PA Signal and TEWL}

We determined whether the PA signal (as an indicator of chemical penetration) was correlated with TEWL (as an indicator of water diffusion) in the stratum corneum. In $A D$ there was a very weak correlation between the PA signals with the hydrophilic dye and TEWL values at 2 minutes $(r=0.309, p=0.002$; Fig. $5 A)$ and between the PA signals with the hydrophilic dye and
TEWL values at 5 minutes $(r=0.224, p=0.026$; Fig. $5 \mathrm{~B})$. In contrast, no correlation existed between the PA signals with the lipophilic dye and TEWL values at 2 minutes ( $r=0.073, p=0.475$; Fig. $5 \mathrm{C}$ ) or between the PA signals with the lipophilic dye and TEWL values at 5 minutes $(r=0.086, p=0.401$; Fig. 5D). These data suggest that the penetration behavior of the hydrophilic dye as measured with PAS is a better reflection 
Red 215 (lipophilic dye)

(A) 2 min dye application

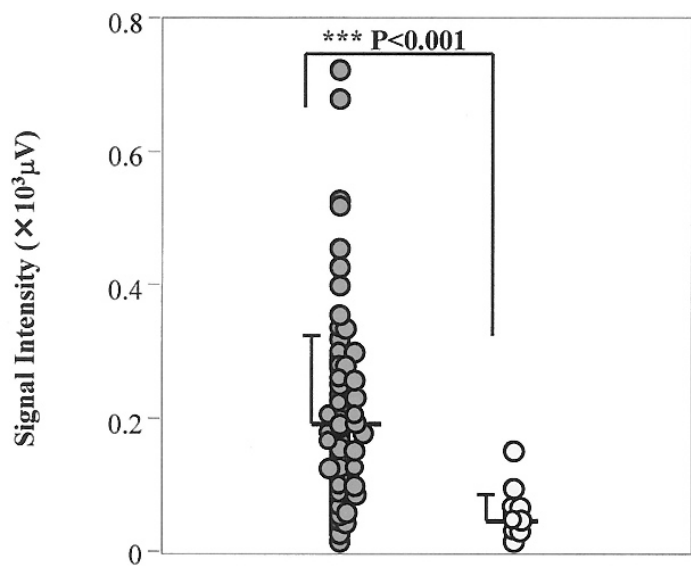

Atopic dermatitis Healthy controls clinically normal skin
(B) 5 min dye application

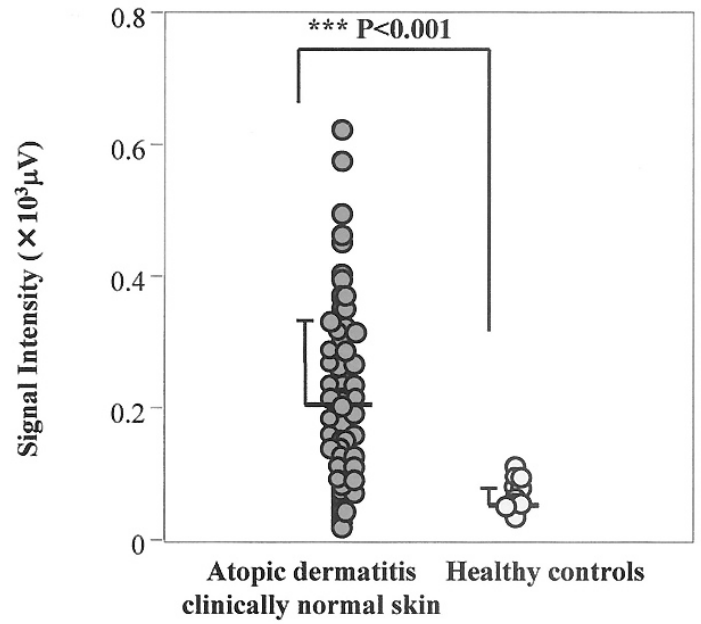

Yellow 4 (hydrophilic dye)

(C)

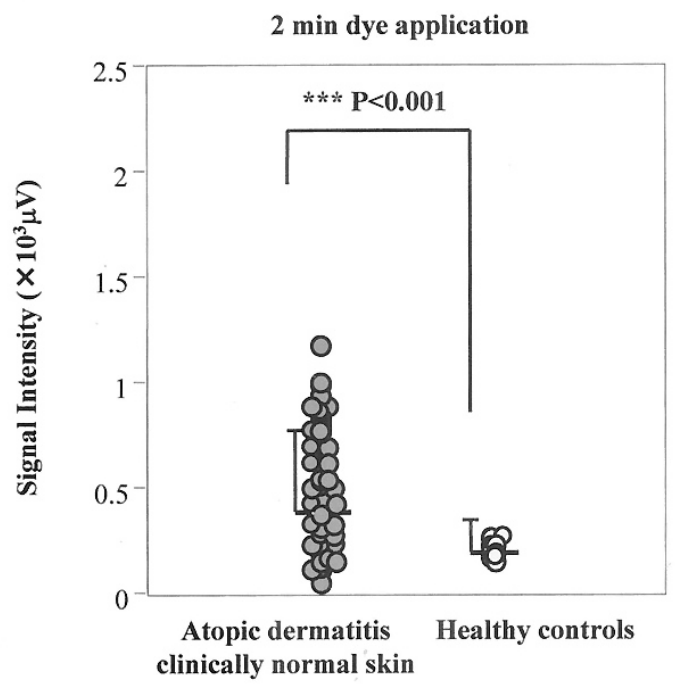

(D)

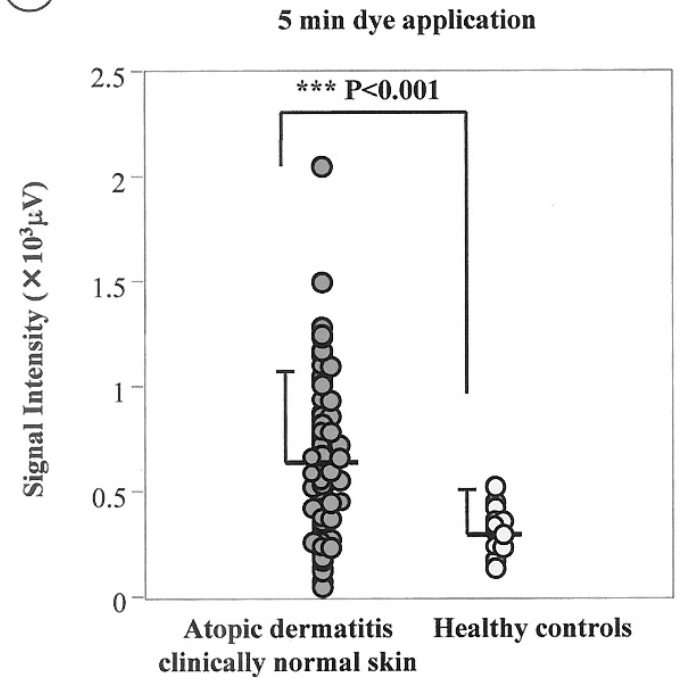

Figure 4.

Penetration rates of lipophilic $(A$ and $B)$ or hydrophilic $(C$ and $D)$ dyes through the stratum corneum. A patch chamber containing $640 \mu$ l of the mixed dye solution (Red 215: $0.12 \mathrm{~mm}$, Yellow 4: $0.06 \mathrm{~mm}$ ) was topically applied for 2 minutes (AD, $n=103$, average age $=26$ years; healthy controls, $n=10$, average age $=35$ years; A and $C$ ) or 5 minutes (AD, $n=100$, average age $=27$ years; healthy controls, $n=10$, average age $=35$ years; $B$ and $D)$ to the forearm skin under closed patch test conditions. Thirty seconds after removal of the patch, the portable open-ended PA cell was attached to the skin surface of the treated sites for subsequent measurements of PA signals.

of TEWL and that measurements of the PAS signal of hydrophilic and lipophilic molecules exhibit different facets of the stratum corneum barrier function.

\section{Relationship Between the PA Signals of Dyes Through the Stratum Corneum and the Severity of $A D$}

The penetration of various chemicals through the stratum corneum is distinct in $A D$ skin compared with normal skin. Therefore, we examined the PA signals of lipophilic and hydrophilic dyes in relation to the severity of AD. The difference between the PA signal of the healthy control group and the mild $A D$ group with the lipophilic dye was significant ( $p<0.05$; Fig. 6, A and $B)$ in contrast to no significant difference with the hydrophilic dye (Fig. 6, C and D). On the other hand, the intensities of the PA signals obtained with the hydrophilic dye differed significantly among the three groups of $A D$ patients with different severity $(p<0.05$ 
(A)

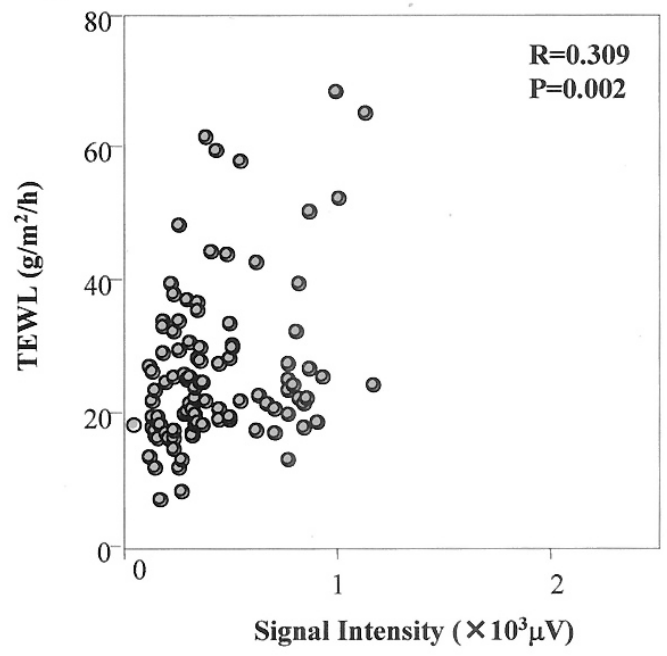

Hydrophilic dye/2 min dye application

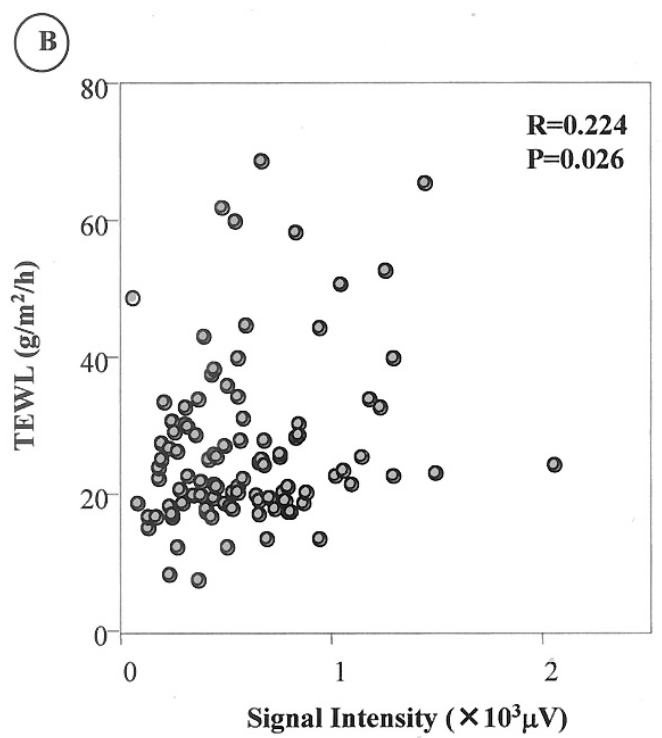

Hydrophilic dye/5 min dye application

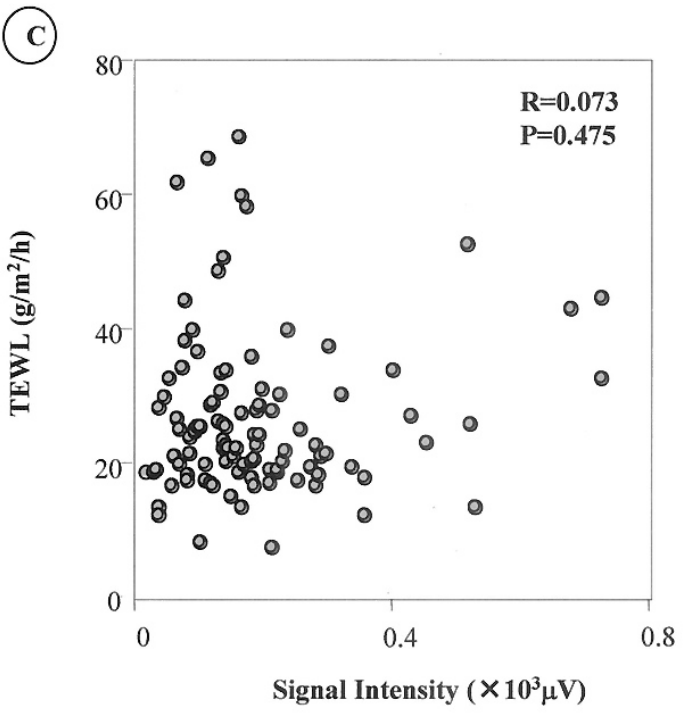

Lipophilic dye/ 2 min dye application
(D)

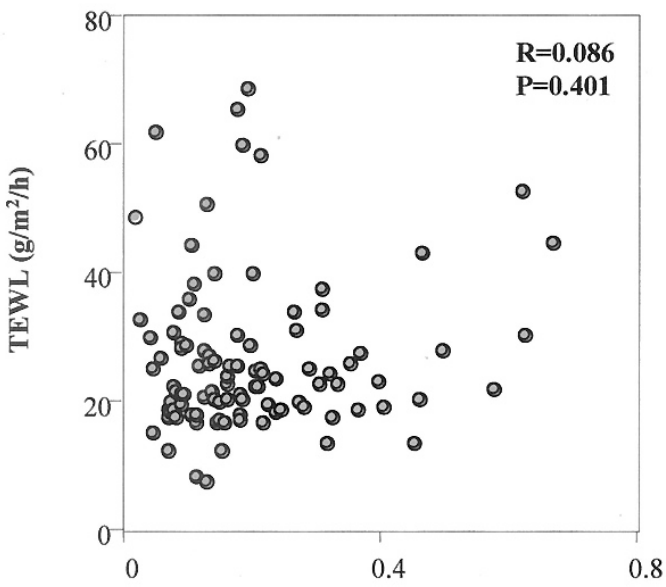

Signal Intensity $\left(\times 10^{3} \mu \mathrm{V}\right)$

Lipophilic dye/ 5 min dye application

\section{Figure 5.}

Relationship between PA signals and TEWL values. PA signals and TEWL were measured as in Figure 4. A, PA signal (at 2 minutes) with the hydrophilic dye/TEWL value (AD, $n=103$, average age $=26$ years). B, PA signal (at 5 minutes) with the hydrophilic dye/TEWL value (AD, $n=100$, average age $=27$ years). $C$, PA signal (at 2 minutes) with the lipophilic dye/TEWL value $(A D, n=103$, average age $=26$ years). D, PA signal (at 5 minutes) with the lipophilic dye/TEWL value (AD, $n=$ 100 , average age $=27$ ).

between the mild and moderate groups and between the mild and severe groups at 2 minutes [Fig. 6C] and $p<0.01$ between the mild and severe groups at 5 minutes [Fig. 6D]). In contrast, there was a significant difference $(p<0.05)$ between only the mild and the moderate groups in the PA signals obtained with the lipophilic dye at 2 minutes (Fig. 6, A). A similar weak correlation for the severity of $A D$ was also observed for TEWL, although there were no significant differences between the mild and moderate groups of $A D$ and healthy controls (Fig. 7). These observations indicate that the signals obtained with the hydrophilic dye are better suited to evaluate the severity of $A D$ than are the signals obtained with the lipophilic dye.

\section{Relationship Between PA Signals and Serum IgE Levels}

Serum IgE levels represent a hallmark of the atopic diathesis, and about $80 \%$ of $A D$ patients have increased levels of serum IgE (Leung, 2000). There was a strong correlation $(n=7, r=0.79, p<0.05$ at 2 minutes; and $n=7, r=0.92, p<0.005$ at 5 minutes) between the intensity of PA signals obtained with the hydrophilic dye (Yellow 4) and serum IgE levels in the 
Red 215 (lipophilic dye)

(A)

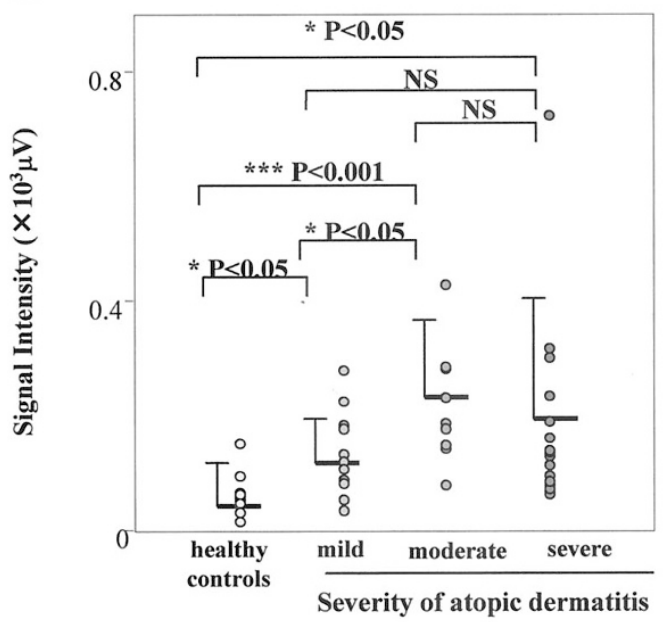

(B)

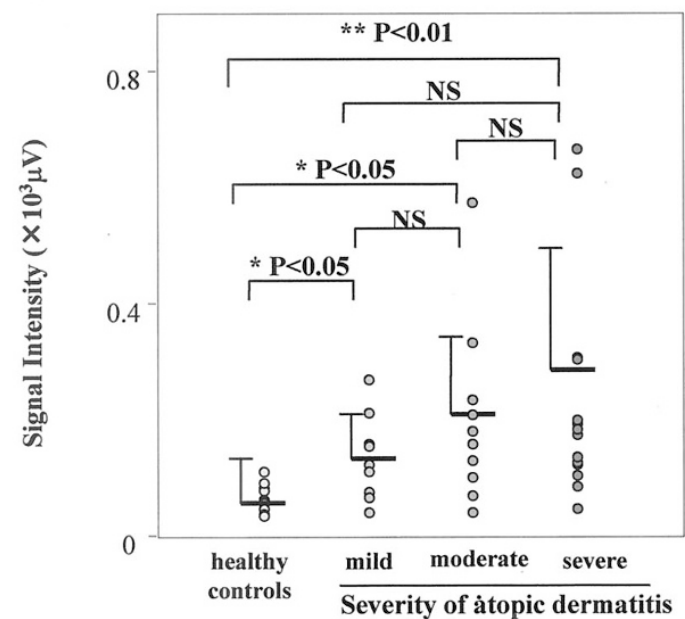

Yellow 4 (hydrophilic dye)

(C)

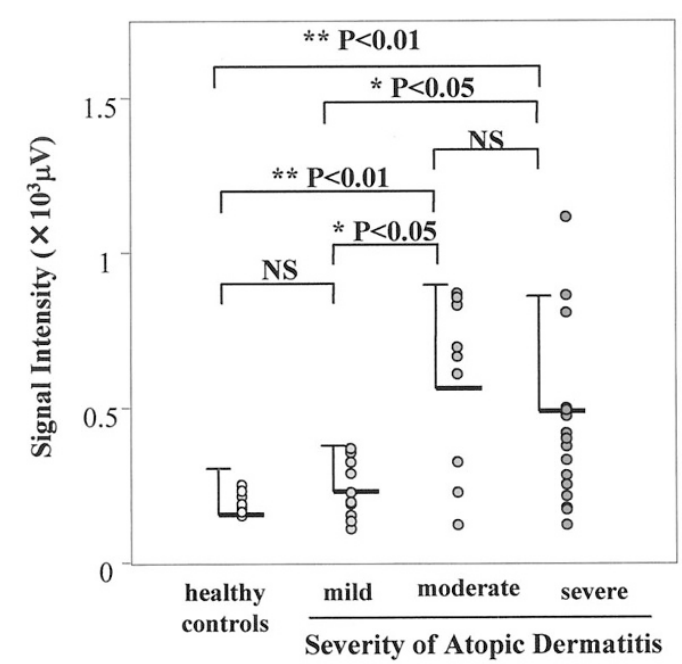

5 min dye application

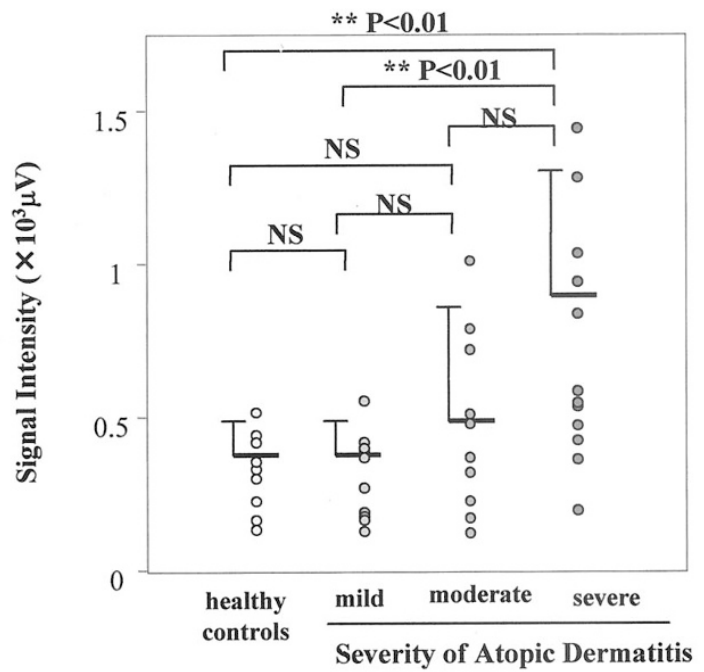

Figure 6.

Relationship between the penetration rates of lipophilic or hydrophilic dyes and the severity of AD. A, Lipophilic dye (Red 215$)$ at 2 minutes (healthy controls, $n=$ 10 , average age $=35$ years; $A D$ mild, $n=6$, average age $=25$ years; $A D$ moderate, $n=5$, average age $=27$ years; $A D$ severe, $n=10$, average age $=28$ years). B, Lipophilic dye (Red 215) at 5 minutes (healthy controls, $n=10$, average age $=35$ years; $A D$ mild, $n=6$, average age $=25$ years; $A D$ moderate, $n=5$, average age $=27$ years; AD severe, $n=10$, average age $=28$ years). C, Hydrophilic dye (Yellow 4) at 2 minutes (healthy controls, $n=10$, average age $=35$ years; $A D$ mild, $n=6$, average age $=25$ years; AD moderate, $n=5$, average age $=27$ years; AD severe, $n=10$, average age $=28$ years). D, Hydrophilic dye (Yellow 4 ) at 5 minutes (healthy controls, $n=10$, average age $=35$ years; $A D$ mild, $n=6$, average age $=25$ years; $A D$ moderate, $n=5$, average age $=27$ years; $A D$ severe $n=10$, average age $=28$ years). The penetration rates of lipophilic $(A$ and $B)$ and hydrophilic $(C$ and $D)$ dyes at $2(A$ and $C)$ and $5(B$ and $D)$ minutes are expressed as the PA signal measured by the PAS after the application of a patch chamber containing a mixed solution of Red 215 and Yellow 4 , at a concentration of 0.12 and $0.06 \mathrm{~mm}$, respectively, to the forearm skin.

severe AD group only (Fig. 8, A and B). In contrast, the intensity of PA signals with the lipophilic dye did not correlate with serum IgE levels in this group (data not shown). The mild and moderate AD groups did not show any correlation between the stratum corneum function and serum IgE levels (data not shown).

\section{Discussion}

In this study, we applied the PAS method for the first time to clarify the status of the in vivo skin barrier functions in AD. The results obtained with the externally applied dyes having different water solubility 


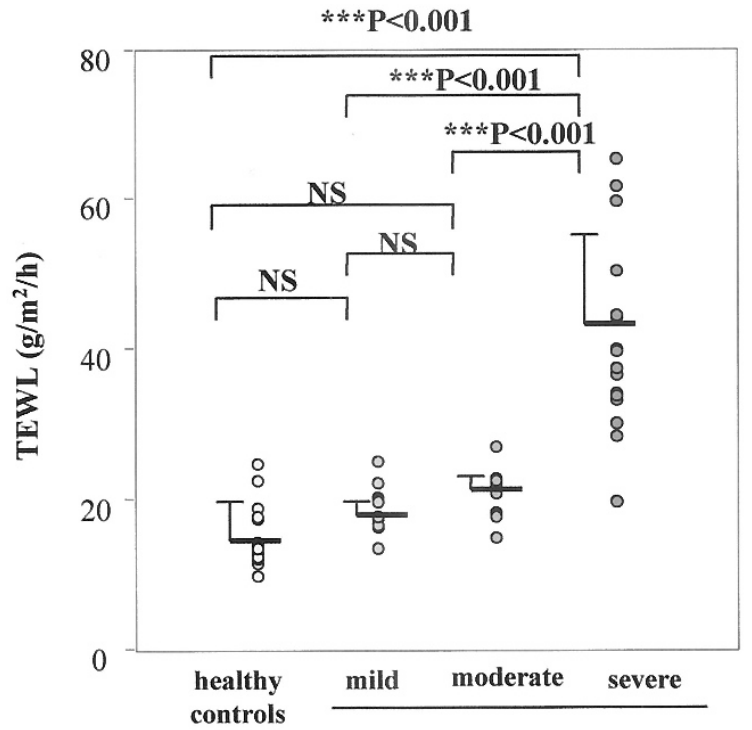

Severity of Atopic Dermatitis

Figure 7.

Relationship between TEWL values and the severity of AD. TEWL from the skin was measured as in Figure 6 (healthy controls, $n=10$, average age $=35$ years; $\mathrm{AD}$ mild, $n=6$, average age $=25$ years; $\mathrm{AD}$ moderate, $n=5$, average age $=27$ years; $A D$ severe, $n=10$, average age $=28$ years).

demonstrated that the stratum corneum of clinically normal skin in patients with $A D$ had a higher permeability rate compared with that of healthy control skin. Since clinically normal skin in patients with $A D$ has minimal or no inflammation (Wakita et al, 1994), this observation strongly suggests that defects in the permeability barrier function in $A D$ are intrinsic and are not associated with inflammatory events. Although there was a significant increase both in PA signals and
2 min dye application

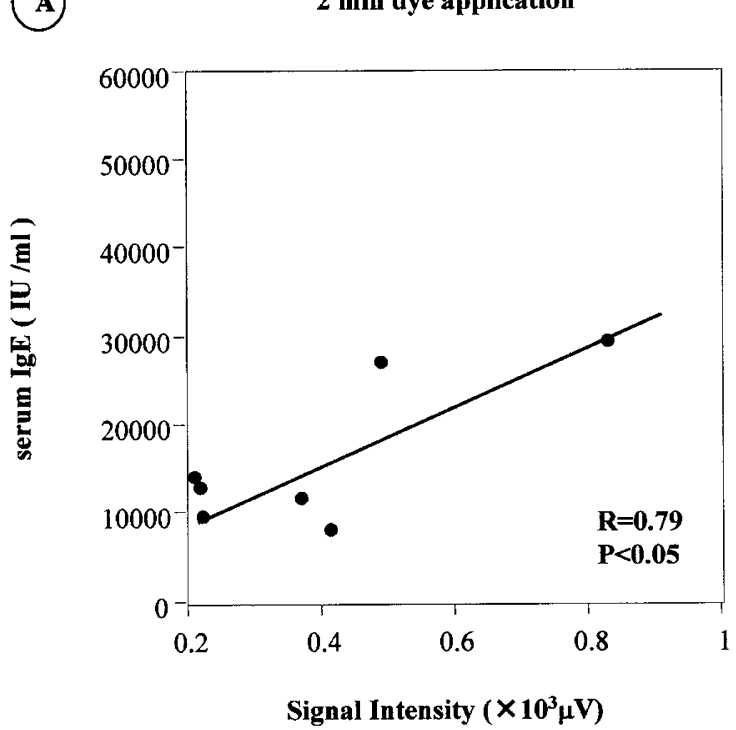

(A) in TEWL values in AD compared with healthy controls, the degree of barrier disruption in $A D$ expressed as a relative ratio to healthy controls was greater with the PAS method (2.98- to 1.8-fold) than with the TEWL measurement (1.73-fold). This indicates that PAS is more useful than TEWL for evaluating the barrier function of skin.

Since AD could be considered a barrier disease in which antigens and irritants that have penetrated (or permeated) the skin trigger and worsen the dermatitis, it is of interest to compare the PA signal for dyes with the severity of AD (according to the criteria of Rajika and Langeland, 1989). Such comparisons revealed that the penetration of the hydrophilic dye through the stratum corneum correlated with the severity grade of AD. In contrast, there was no significant difference in the penetration rate of the lipophilic dye among the three AD groups, thus suggesting that there was no association of lipophilic penetration with the severity of that disease. This may support the notion that molecules with hydrophilic rather than lipophilic properties predominantly elicit and worsen skin inflammation in $A D$ patients. Furthermore, the severity of disease seems to be determined in part by the amounts of irritants and allergens that have penetrated the stratum corneum. This is consistent with the clinical evidence that patients with severe $A D$ have higher levels of IgE specific for house dust and mite antigens than do those patients with mild and moderate $A D$ (Uehara, 1986). In those antigens, proteins are presumed to be solubilized by sweat and to then easily penetrate the stratum corneum in patients with AD. There is a large published literature assigning house dust or mite antigens, and metals, as possible pathogenic allergens and accelerators, respectively, for $A D$ (Cooper, 1994; Deleuran et al, 1998; Fitzharris and
(B)

5 min dye application

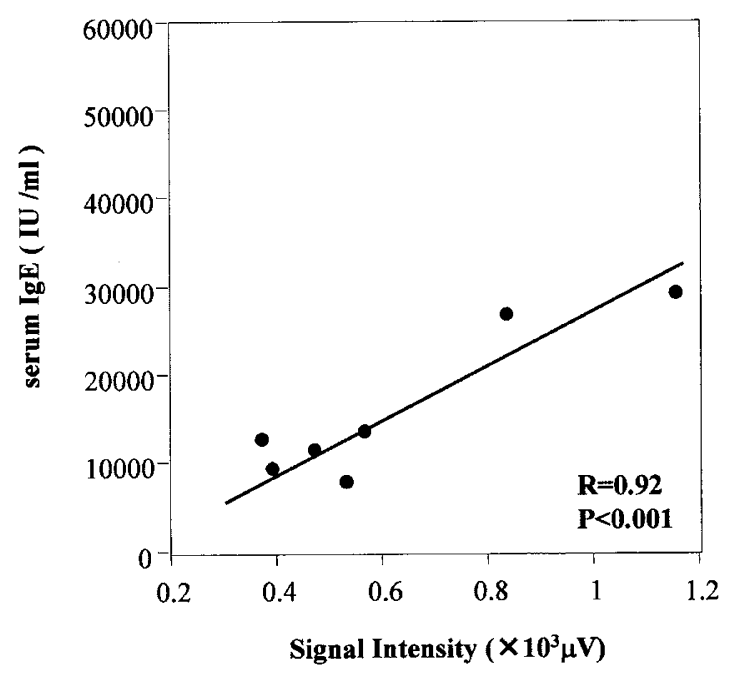

Figure 8.

Correlation between the penetration rates of the hydrophilic dye (Yellow 4) and serum IgE Levels. The penetration rates of the hydrophilic dye at 2 (A) and 5 (B) minutes is expressed as in Figure 4. Serum IgE levels were measured by ELISA using peripheral blood of patients with AD. Severe AD group, $n=7$, average age $=27$ years. 


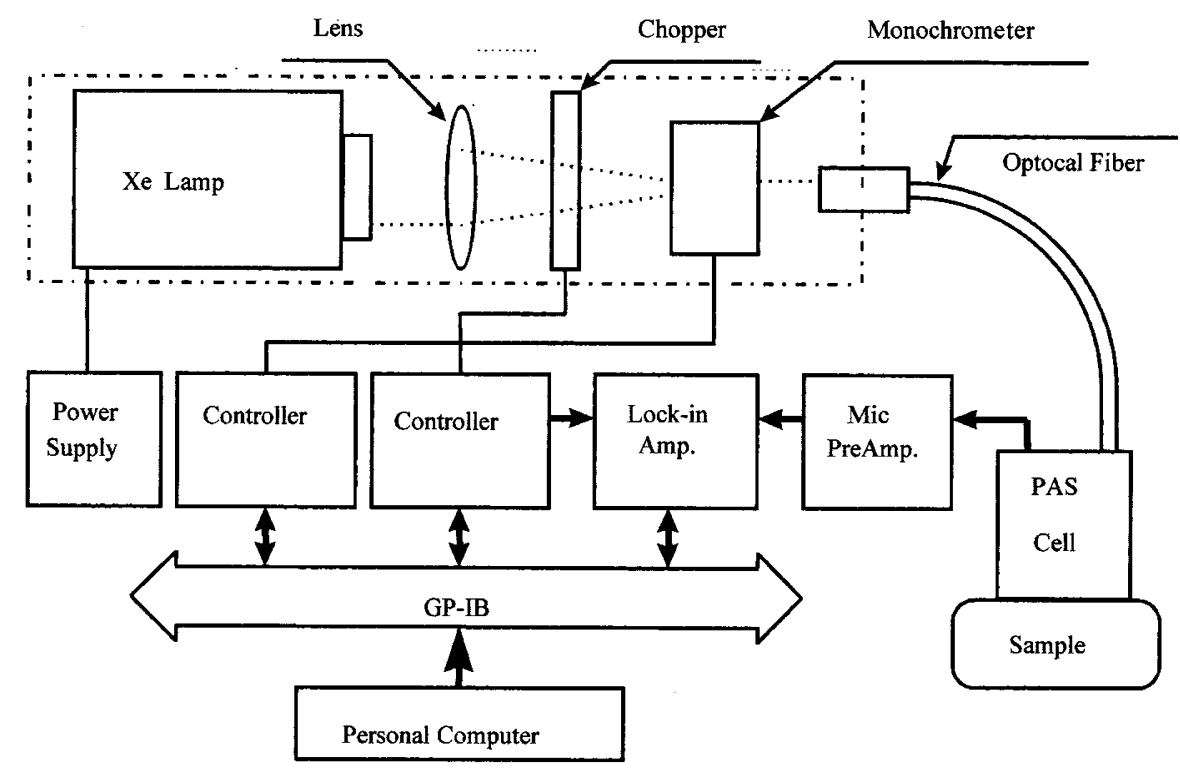

Figure 9.

Diagram of the PAS with an open-ended PA cell. The PAS consists of an Xe lamp, lamp housing, power supply, lens, chopper/wheel, chopper controller, monochrometer/grating, monochrometer controller, optical fiber, lock-in-amp, microphone, microphone pre-amp, and microphone power supply.

Window $\left(\mathrm{SiO}_{2}\right)$

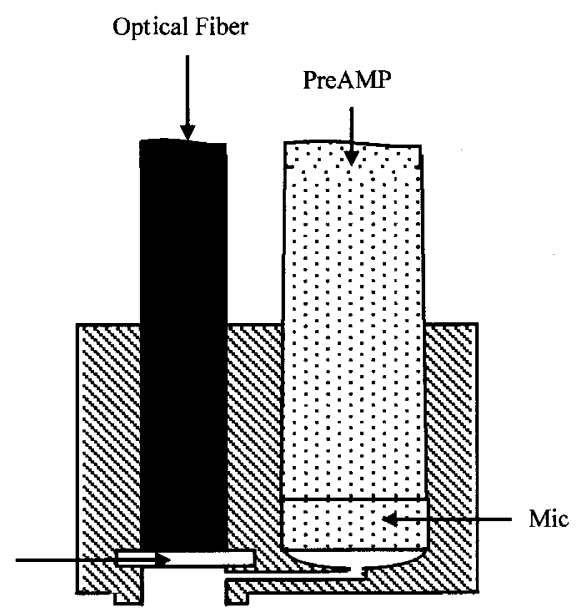

Figure 10.

Diagram of portable open-ended PA cells for in vivo measurement on skin. After application of the portable open-ended PA cell to the forearm skin, the PA signal detected by the cell within approximately a $14-\mu \mathrm{m}$ depth from the skin surface was analyzed using a lock-in amplifier (NF, 6610A) and was recorded using a chart recorder.

Riley, 1999; Gloor et al, 1981; Ogawa and Yoshiike, 1993; Rothe and Grant-Kels, 1996; Tan et al, 1996; Tanaka et al, 1994; Tupker et al, 1990). In accordance with the evaluation by PAS using the hydrophilic dye, TEWL values were significantly accentuated only in the severe $A D$ group. Our data showed no strong correlations between the PA signals obtained with hydrophilic or with lipophilic dyes and TEWL values in $\mathrm{AD}$ patients and healthy controls, which suggests that each value represents a different barrier function against different substances, including water molecules. Furthermore, estimation of the barrier function by TEWL values would provide false results in terms of evaluating the penetration of lipophilic substances.
Collectively, these findings suggest that the barrier function of the skin as assessed with hydrophilic substances reflects the severity of $A D$, whereas the barrier function against lipophilic substances might provide a diagnostic aid for mild AD.

It has been reported that serum IgE levels correlate with the severity of AD (Beck and Leung, 2000; Leung, 2000; Schafer et al, 1999; Uehara, 1986). The distinct relevance of the hydrophilic barrier function to the severity of $A D$ allowed us to determine whether the PAS signal obtained with the hydrophilic dye is associated with the serum IgE level. Interestingly, we found that there was a close relationship $(r=0.787$ at 2 minutes, $r=0.915$ at 5 minutes) between PAS signals and serum IgE levels in the severe AD group, whereas no relationship existed among other parameters, including TEWL values, in the mild or moderate groups of AD. In a previous study (Kondo et al, 1998), we found that allergic reactions induced percutaneously with mite antigens are dominated by $\mathrm{T}$ helper 2-type immunity and lead to increased serum lgE levels in mice. Taken together, the close relationship between the hydrophilic barrier function and the serum IgE level in the severe $A D$ group suggests that $A D$ patients who have profound dysfunction of the hydrophilic barrier may be highly susceptible to percutaneous sensitization by the more water-soluble allergens derived from environmental antigens such as house dust mites. This would then result in increased serum lgE levels as a result of inflammatory reactions specific for observed allergens.

In conclusion, the present study demonstrates that a variety of barrier functions are disclosed by PAS in conjunction with the use of molecules having different water solubility. Furthermore, our data indicate for the first time that the clinically normal skin of patients with 


\section{Red 215 \\ (Lipophilic dye)}

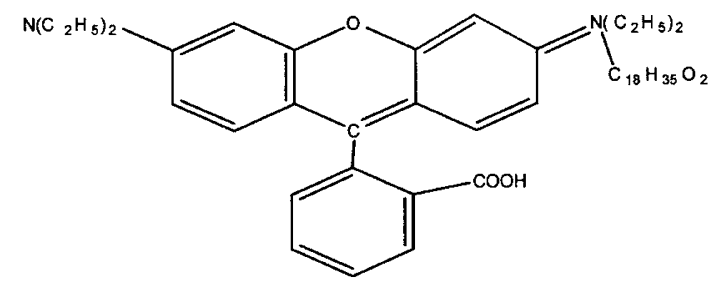

\section{Molecular Weight 423}

\section{Solubility $0.2 \%$ (ethanol)$$
0.2 \% \text { (water) }
$$

Figure 11.

Chemical structure and solubility of Red 215 and Yellow 4.

$A D$ has a potential impairment in the in vivo cutaneous permeability barrier against both lipophilic and hydrophilic reagents. Those abnormal barrier functions may predispose the skin to inflammatory processes evoked by irritants and allergens in AD.

\section{Methods}

\section{Diagnosis}

A total of 27 patients with AD (aged 19-44 years; 10 men, 17 women), and 18 healthy non-AD volunteers with low serum IgE levels (less than $250 \mathrm{U} / \mathrm{ml}$ ) (aged 20-56 years; 7 men, 11 women) were enrolled in this study. The diagnosis of AD was made according to the criteria of Hanifin and Rajika (1989). The severity of AD was evaluated by the criteria reported by Rajika and Langeland (1989) and was classified into mild, moderate, or severe. Serum IgE levels of the patients ranged from 7670 to $53300 \mathrm{lU} / \mathrm{ml}$ and averaged 18254 $\mathrm{IU} / \mathrm{ml}$. All patients were referred by previous doctors who prescribed their treatment schedules. None had taken oral steroids, and none had evident systemic disease. Measurements in this study were performed after informed consent had been obtained.

\section{Measurement Sites of the Skin}

The volar surface of forearm skin was principally subjected to all measurements. In AD, clinically normal areas on the volar site were chosen to measure the PA signal, TEWL, and conductance. The patients did not apply any ointment or cream on the examined sites for at least 5 days before the measurements. Concerning

\section{Yellow 4 \\ (Hydrophilic dye)}

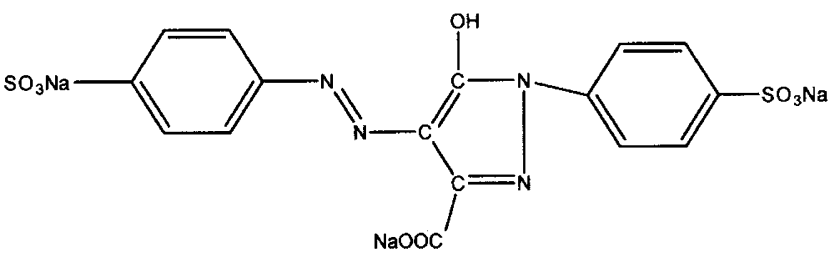

\section{Molecular Weight 524 Solubility $0.08 \%$ (ethanol) \\ $0.5 \%$ (water)}

the inflammatory state at the clinically normal tested sites, we have previously demonstrated that clinically normal skin from patients with AD has no histologic evidence of inflammation (Wakita et al, 1994), suggesting that in the current study, no substantial inflammatory changes were present in the nonlesional skin.

\section{TEWL and Water-Holding Property}

TEWL was measured by detecting evaporated water according to a modified method (Gfesser et al, 1996; Nilsson, 1977) using a crystal vibration microbalance system (Endo et al, 2001) and is expressed as $\mathrm{g} / \mathrm{m}^{2} / \mathrm{hr}$. The amount of skin surface water was evaluated by an impedance meter (Werner, 1986; Yoshizuka et al, 1983) and is expressed as conductance ( $\mu \mathrm{mho})$.

\section{PAS}

A PAS with an open-ended PA cell (Takamoto et al, 1992, 1994) was established in our laboratories (Shioya et al, 2001), as shown in Figure 9. The system consists of an Xe lamp (L2274; Hamamatsu Photonics, Hamamatsu, Japan); lamp housing (no. 66005; Oriel, Stratford, Connecticut); power supply (no. 68805; Oriel); lens (no. 66xxx, 3 inch F0.7; Oriel); chopper/wheel (no. 75152/no. 75166; Oriel); chopper controller (no. 70103; Oriel); monochrometer/grating (no. 77298/no. 77919), monochrometer controller (no. 77325, no. 20050), optical fiber (OF160-1.5 type; Tokyo Instruments); lock-in-amp (5610B; NF circuit block); microphone (4166; B\&K, Alleroed, Denmark); microphone pre-amp (4166; B\&K); and microphone power supply (5935; B\&K). A diagram of the portable 
open-ended PA cell for in vivo measurements of the skin is shown in Figure 10. After the portable openended PA cell was attached to each measurement site, the PA signal detected by the cell (within approximately $14-\mu \mathrm{m}$ depth from the skin surface) was analyzed using a lock-in amplifier (NF, 6610A, Kanagawa, Japan) and was recorded using a chart recorder. The wavelength of the light source was adjusted to 550 for Red 215 and to $425 \mathrm{~nm}$ for Yellow 4. The PA signal is expressed as microvolts $(\mu \mathrm{V})$ for the short time measurement study or as a standardized signal intensity for the time course study calculated as follows: standardized signal intensity = observed signal intensity - signal intensity before application/initial signal intensity - signal intensity before application.

\section{Sample Application and Measurements}

We used Red 215 and Yellow 4 (chemical structures are depicted in Fig. 11) as reagents for the penetration measurement, which are lipophilic and hydrophilic, respectively. Red 215 and Yellow 4 were solubilized in a solution of $95 \%$ ethanol and $5 \%$ water at concentrations of 0.18 to 0.23 and 0.064 to $0.150 \mathrm{~mm}$, respectively. For the time course study, each solution containing Red 215 at $0.22 \mathrm{~mm}$ and Yellow 4 at 0.15 $\mathrm{mm}$ was topically applied to the forearm skin. For the short time measurement study, a patch chamber containing $640 \mu \mathrm{l}$ of the solution (containing both Red 215 at $0.118 \mathrm{~mm}$ and Yellow 4 at $0.06 \mathrm{~mm}$ ) was topically applied for 2 or 5 minutes to the forearm skin under closed patch test conditions. The patches were removed before measurements were taken.

For determining the distribution of dyes within the stratum corneum, 95\% ethanol solutions containing Red 215 at $0.22 \mathrm{~mm}$ and Yellow 4 at $0.15 \mathrm{~mm}$ were applied for 2 minutes to the forearm. Measurements were taken after each step of tape stripping that was repeated 15 times. For measurements, excess solution was wiped off with a towel, and 30 seconds later, the portable open-ended PA cell was attached to the dye-treated skin surface.

\section{Statistics}

The level of significance of differences was calculated by the Student's $t$ test.

\section{References}

Beck LA and Leung DY (2000). Allergen sensitization through the skin induces systemic allergic responses. J Allergy Clin Immunol 106:S258-S263.

Broberg A, Faergemann J, Johansson S, Johansson SG, Strannegard IL, and Svejgaard E (1992). Pityrosporum ovale and atopic dermatitis in children and young adults. Acta Derm Venereol 72:187-192.

Cooper KD (1994). Atopic dermatitis: Recent trends in pathogenesis and therapy. J Invest Dermatol 102:128-137.

Deleuran M, Ellingsen AR, Paludan K, Schou C, and Thestrup-Pedersen K (1998). Purified Der p1 and p2 patch tests in patients with atopic dermatitis: Evidence for both allergenicity and proteolytic irritancy. Acta Derm Venereol 78:241-243.

Endo K, Suzuki N, Hoshi M, Shioya Y, Kato T, and Fujikura Y (2001). The evaluation of epoxy resin coated quartz crystal humidity sensor and the measurement of water evaporation from human surface. Surface Technol 52:54-58.

Fitzharris P and Riley G (1999). House dust mites in atopic dermatitis. Int J Dermatol 38:173-175.

Gfesser M, Rakoski J, and Ring J (1996). The disturbance of epidermal barrier function in atopy patch test reactions in atopic eczema. Br J Dermatol 135:560-565.

Gloor M, Heymann B, and Stuhlert TH (1981). Infraredspectroscopic determination of the water content of the horny layer in healthy subjects and in patients suffering from atopic dermatitis. Arch Dermatol Res 271:429-436.

Hara J, Higuchi K, Okamoto R, Kawashima M, and Imokawa G (2000). High-expression of sphingomyelin deacylase is an important determinant of ceramide deficiency leading to barrier disruption in atopic dermatitis. J Invest Dermatol 115:406-413.

Hanifin JM and Rajika G (1989). Diagnostic features of atopic dermatitis. Acta Derm Venereol 92:44-47.

Higuchi K, Hara J, Okamoto R, Kawashima M, and Imokawa $G$ (2000). The skin of atopic dermatitis patients contains a novel enzyme, glucosylceramide sphingomyelin deacylase, which cleaves the $\mathrm{N}$-acyl linkage of sphingomyelin and glucosylceramide. Biochem J 350:747-756.

Imokawa G (1993). Water and the stratum corneum. In: $P$ Elsner, E Berardesca, HI Maibach, editors. Bioengineering of skin: In vitro and in vivo models, vol. 1, chap. 3. Boca Raton: CRC Press, 23-47.

Imokawa G (1999). Skin moisturizers: Development and clinical use of ceramides. In: Loden M , editor. Dry skin and moisturizers. Boca Raton: CRC Press, 269-299.

Imokawa G, Abe A, Jin K, Higaki Y, Kawashima M, and Hidano A (1991). Decreased level of ceramides in stratum corneum of atopic dermatitis: An etiologic factor in atopic dry skin? J Invest Dermatol 96:523-526.

Imokawa G, Yada Y, Higuchi K, Okuda M, Ohashi Y, and Kawamata A (1994). Pseudo-acylceramide with linoleic acid produces selective recovery of diminished cutaneous barrier function in essential fatty acid-deficient rats and has an inhibitory effect on epidermal hyperplasia. J Clin Invest 94:89-96.

Kondo H, Ichikawa $\mathrm{Y}$, and Imokawa G (1998). Percutaneous sensitization with allergens through barrier-disrupted skin elicits a Th2-dominant cytokine response. Eur J Immunol 28:769-779.

Lammintausta K, Kalimo K, and Viander M (1993). Candida albicans and atopic dermatitis. J Clin Exp Allergy 23:332339.

Leung DY (2000). Atopic dermatitis: New insights and opportunities for therapeutic intervention. J Allergy Clin Immunol 105:860-876.

Murata Y, Ogata J, Higaki Y, Kawashima M, Yada Y, Tsuchiya T, Kawaminami S, and Imokawa G (1996). Abnormal expression of sphingomyelin acylase in atopic dermatitis: An etiologic factor for ceramide deficiency? J Invest Dermatol 106:1242-1249. 
Nilsson GE (1977). Measurement of water exchange through skin. Med Biol Eng 15:209-218.

Ogawa $\mathrm{H}$ and Yoshiike $\mathrm{T}$ (1993). A speculative view of atopic dermatitis: Barrier dysfunction in pathogenesis. J Dermatol Sci 5:197-204.

Rajika G and Langeland T (1989). Grading of the severity of atopic dermatitis. Acta Derma Venereol Suppl 144:13-14.

Rothe MJ and Grant-Kels JM (1996). Atopic dermatitis: An update. J Am Acad Dermatol 35:1-13.

Schafer T, Heinrich J, Wjst M, Adam H, Ring J, and Wichmann ME (1999). Association between severity of atopic eczema and degree of sensitization to aeroallergens in school children. J Allergy Clin Immunol 104:1280-1284.

Shioya Y, Kokubo K, Sato M, and Fujikura Y (2001). In vivo evaluation of percutaneous absorption by photoacoustic method: Development of practical apparatus and its application to human skin. J Jap Cosmet Sci Soc 25:67-73.

Takamoto R, Namba R, Matsuoka M, and Sawada T (1992). Human in vivo percutaneous absorptiometry using the laserphotoacoustic method. Anal Chem 64:2661-2663.

Takamoto R, Yamamoto S, Namba R, Takamatsu T, Matsuoka M, and Sawada T (1994). In vivo percutaneous absorptiometry by a laser photoacoustic method using a novel open-ended cell combined with light guide. Anal Chem 66:2267-2271.

Tanaka M, Aiba S, Matsumura N, Aoyama H, Tabata N, Sekita Y, and Tagami H (1994). IgE-mediated hypersensitivity and contact sensitivity to multiple environmental allergens in atopic dermatitis. Arch Dermatol 130:1393-1401.
Tan BB, Weald D, Strickland I, and Friedmann PS (1996). Double-blind controlled trial of effect of housedust-mite allergen avoidance on atopic dermatitis. Lancet 6:15-18.

Tupker RA, Pinnagoda J, Coenraads PJ, and Nater JP (1990). Susceptibility to irritants: Role of barrier function, skin dryness and history of atopic dermatitis. Br J Dermatol 123:199205.

Uehara M (1986). Heterogeneity of serum IgE levels in atopic dermatitis. Acta Derm Venereol 66:404-408.

Wakita H, Sakamoto T, Tokura Y, and Takigawa M (1994). E-selectin and vascular cell adhesion molecule- 1 as critical adhesion molecules for infiltration of $\mathrm{T}$ lymphocytes and eosinophils in atopic dermatitis. J Cutan Pathol 21:33-39.

Watanabe M, Tagami H, Horii I, Takahashi M, and Kligman AM (1991). Functional analysis of the superficial stratum corneum in atopic xerosis. Arch Dermatol 127:1689-1692.

Werner $Y$ (1986). The water content of the stratum corneum in patients with atopic dermatitis: Measurement with the corneometer CM 420. Acta Derm Venereol 66:281-284.

Wood LC, Elias PM, Calhoun C, Tsai JC, Grunfeld C, and Feingold KR (1996). Barrier disruption stimulates interleukin$1 \&$ fnof; $i$ expression and release from a pre-formed pool in murine epidermis. J Invest Dermatol 106:397-403.

Yoshizuka N, Suganuma T, Imokawa G, Imamura T, Okamoto K, and Misumi H (1983). A simplified method for detecting skin surface lipids and skin surface hydration. The Nishinihon J Dermatol 45:595-601. 